Voix et Images

voixetimages

\title{
Écrire ce qui donne à lire. Nicole Brossard lectrice
}

\section{Robert Dion}

Volume 17, numéro 2 (50), hiver 1992

L’âge de la critique, 1920-1940

URI : https://id.erudit.org/iderudit/200960ar

DOI : https://doi.org/10.7202/200960ar

Aller au sommaire du numéro

\section{Éditeur(s)}

Université du Québec à Montréal

\section{ISSN}

0318-9201 (imprimé)

1705-933X (numérique)

Découvrir la revue

Citer cet article

Dion, R. (1992). Écrire ce qui donne à lire. Nicole Brossard lectrice. Voix et Images, 17(2), 250-263. https://doi.org/10.7202/200960ar
Résumé de l'article

Résumé

À tort ou à raison, Nicole Brossard a été considérée comme la principale théoricienne québécoise de la 'nouvelle écriture -, puis de l'écriture des femmes. Le présent article s'attache à retracer une pensée littéraire dont l'influence s'exerce au Québec depuis plus de 25 ans. Ont fait l'objet d'une attention particulière les textes qui tentent d'intégrer au projet d'écriture de la modernité quelques consistantes propositions de lecture. Il s'agit ici d'envisager l'activité de Nicole Brossard comme lectrice du projet moderne des deux dernières décennies, de manière à dégager son apport à la théorisation de la -nouvelle écriture: d'utilisation que vous pouvez consulter en ligne.

https://apropos.erudit.org/fr/usagers/politique-dutilisation/ 


\title{
Écrire ce qui donne à lire. Nicole Brossard lectrice
}

\section{Robert Dion,: Université du Québec à Rimouski}

\begin{abstract}
À tort ou à raison, Nicole Brossard a été considérée comme la principale théoricienne québécoise de la .nouvelle écriture", puis de l'écriture des femmes. Le présent article s'attache à retracer une pensée littéraire dont l'influence s'exerce au Québec depuis plus de 25 ans. Ont fait l'objet d'une attention particulière les textes qui tentent d'intégrer au projet d'écriture de la modernité quelques consistantes propositions de lecture. Il s'agit ici d'envisager l'activité de Nicole Brossard comme lectrice du projet moderne des deux dernières décennies, de manière à dégager son apport à la théorisation de la " nouvelle écriture .
\end{abstract}

À partir des années soixante, au Québec, le rapport entre écrivains et critiques universitaires s'est radicalement modifié. Ce changement s'est d'abord fait sentir chez les critiques, dont les travaux ont perdu le caractère prescriptif ou condescendant qu'ils avaient pu avoir auparavant. On conviendra qu'une critique du type de celle de $M^{r r}$ Camille Roy, en 1960, n'était plus concevable. C'est que les décennies soixante et soixante-dix ont été marquées par la revalorisation de l'activité d'écrivain, sans doute liée à celle de la littérature québécoise dans son ensemble. Par ailleurs, dans le champ de la théorie et de la critique littéraires - autrefois occupé par les seuls universitaires -, les écrivains ont réussi une percée significative et se sont emparés d'une part importante du discours sur les œuvres. Pendant ce temps, il est vrai, de nombreux professeurs d'université devenaient écrivains, contribuant par là même à l'instauration d'un cumul généralisé des deux fonctions.

Dans le domaine de la critique littéraire ${ }^{1}$, on remarque que les écrivains ont commencé à s'imposer dans le champ littéraire et à

1. Cette étude appartient à une série consacrée à L'Adaptation des modèles théoriques étrangers dans la critique littéraire québécoise (1950-1980). Ce projet 
entrer en compétition avec les professeurs d'université pour le monopole du discours autorisé sur la littérature ${ }^{2}$. Comme l'affirmait à juste titre Madeleine Gagnon dans un colloque "mixte" (professeurs et écrivains) tenu à Bruxelles en 1985, cette génération d'écrivains s'est préoccupée aussi bien de la production des œuvres que de la production du discours d'accompagnement:

Une génération où les discours sur la modernité sont si présents que parfois ils occupent toute la place, le champ de l'écriture. Ils se disent dans .l'œuvre de fiction. en train de s'élaborer, ils s'enoncent dans ce qui se nomme tout de même - roman - ou : poème., Ils disent outre-passer les genres, les genres littéraires traditionnels, anciens, et ils s'appellent finalement :textes'. J'appartiens à une génération où des poètes ou des romanciers expliquent eux-mêmes aux critiques, ou aux lecteurs, ce qu'est la : textualisation $\cdot 3$.

Ce discours d'accompagnement a pu prendre la forme d'articles séparés ou, au contraire; s'inscrire dans les œuvres littéraires ellesmêmes. En règle générale, les professeurs-écrivains '(Pierre Nepveu, Jacques Brault, André Gervais,-par exemple) paraissent avoir privilégié la dissociation œuvres de création/critique (encore que cette dissociation puisse être plus ou moins radicale selon les cas), tandis que les écrivains stricto sensu (qui, parce qu'ils publient surtout des textes de création, sont d'abord connus comme tels, et qui, souvent, enseignent dans les collèges) ont eu plutôt tendance à inclure la théorie au cœur même des œuvres et ont théorisé cette tendance dans des "fictions / théories" ou encore dans des "textes ${ }^{4}$. Si les premiers ont surtout

de recherche est subventionné par le CRSH du Canada et.l'Université du Québec à Rimouski.

2. En France aussi, l'écrivain a investi le discours théorique sur la littérature, l'exemple de Tel Quel s'avérant sans doute le plus spectaculaire à cet égard; toutefois, dans ce pays où le sacre de l'écrivain avait eu lieu depuis longtemps, le phénomène inverse était beaucoup plus remarquable: dans les années soixante, ce sont les critiques tels que Roland Barthes, Julia Kristeva, Hélène Cixous et d'autres qui entrent en compétition avec l'écrivain.

3. Madeleine Gagnon, -Parler l'écriture ou parler la parole •, Modernité/Postmodernité du roman contemporain, sous la direction de Madeleine Frédéric et Jacques Allard, Montréal, Université du Québec à Montréal, coll. . Cahiers du Département d'études littéraires :, $\mathrm{n}^{\circ} 11,1987$, p. 15-22.

4. Jean Yves Collette, cité par Jean Royer: "La modernité québécoise a inventé, sans même y penser, le concept de 'TEXTE comme genre littéraire. [...]. Le 'TEXTE c'est la conscience expérimentale du langage dans un système de production. [...]: Tout ce qui n'est pas 'TEXTE n'est pas moderne et continue de renforcer les genres de la tradition. • (-À Montréal: la Modernité ?, La Nouvelle Barre du jour, $\mathrm{n}^{\circ} 141,1984$, p. 89) Cette revendication du * texte - comme genre littéraire nouveau exerce visiblement une fonction normative qui, comme le remarquait Philippe Lejeune dans Le Pacte autobiographique (Paris, Seuil, 1975), signale un processus institutionnel de redistribution des horizons d'attente. 
contribué à l'étude des "classiques" ou des "nouveaux classiques" québécois (Nepveu sur Victor-Lévy Beaulieu et sur Nicole Brossard, Brault sur Gaston Miron, Gervais sur Nelligan), bref, à l'étude des textes d'autres écrivains, les seconds, en revanche, ont d'abord réfléchi à leur propre pratique de création - ce qui semble, au demeurant, caractéristique des pratiques théoriques autodidactes. Ceux-ci ont procédé, comme l'écrit Roger Chamberland, «à une autothéorisation qui confirme le texte dans le procès de signifiance (Kristeva) et le fixe dans un modèle littéraire, celui du formalisme $[. . .]^{5}$. S'est donc constitué un discours savant marginal, typique des écrivains, qui s'est implicitement donné pour tâche de déjouer le discours universitaire classant (typologisant/historicisant) par l'émiettement du discours théorique à l'intérieur même du texte ${ }^{6}$, battant ainsi en brèche l'idéologie du discours théorique unitaire, de même que l'idée traditionnelle d'^œuvre.

Nicole Brossard est sans doute l'écrivaine formaliste et moderniste par excellence 7 , dans la mesure où elle ne conçoit le texte que forclos dans et par sa théorie. On sait que le discours savant, chez Brossard, n'existe pas de manière autonome, c'est-à-dire découplé d'une pratique d'écriture fictive/théorique, pour reprendre ses propres termes. Ainsi, dans le numéro de La Nouvelle Barre du jour qui a pour titre Onze Analyses (1973) et qui est consacré à des analyses structurales de poèmes québécois, Nicole Brossard s'est contentée de signer le liminaire, d'ailleurs ironiquement intitulé "Vague de précision ${ }^{8}$. Elle n'a fait paraître, ces dernières années, qu'un seul texte de fond qui ne porte pas sur son œuvre et qui est la transcription d'une conférence sur Djuna Barnes ${ }^{9}$, de facture au reste traditionnelle. C'est dire que l'apport de Nicole Brossard à la critique et à la théorie littéraire "pures" (si cette expression a un sens) est mince ${ }^{10}$. Les conférences, les entrevues, les textes de circonstance ${ }^{11}$

5. Roger Chamberland, "Circa les Herbes rouges •, thèse de Ph.D., Québec, Université Laval, 1987, f. 3.

6. Ibid., f. 16.

7. Et ce, d'autant plus que l'écriture est sa principale activité.

8. Nicole Brossard, = Vague de précision , La Barre du jour, $\mathrm{n}^{*} 38-41$, printemps-été 1973, p. 3.

9. Id., - Djuna Barnes de profil moderne , Mon béroïne: les lundis de l'bistoire des femmes. An I, conférences du Théâtre expérimental des femmes, 1980-1981, Montréal, Éditions du Remue-ménage, 1981, p. 189-214.

10. On ne compte pas ici pour une contribution significative les recensions qu'elle a pu donner, au tout début de sa carrière, au Quartier latin.

11. Textes dont quelques-uns sont repris dans Double Impression. Poèmes et textes 1967-1984 (Montréal, Éditions de l'Hexagone, 1984), et dans La Lettre aérienne (Montréal, Éditions du Remue-ménage; 1985). Textes qui s'écrivent entre deux livres et qui sont, note Brossard, *une ponctuation qui reflète le livre antérieur ou 
dont il sera question dans la présente étude n'ont pour objet que ses livres ou sa pratique d'écriture; lectrice, Nicole Brossard l'est avant tout d'elle-même. Toute son activité commentatrice vise à se rendre lisible à soi-même, intégralement; il s'agit, en définitive, d'amorcer un obstiné travail de lecture, une patiente traversée des signes. C'est cet aspect du travail de Brossard, centré sur la lecture et surtout sur la relecture (puisque "la plupart des gens du texte savent se relire à temps ${ }^{12}$ ), qui nous intéressera d'abord ici. Nous tenterons, d'une part, de déterminer la place de la lecture dans son ouvre, et, d'autre part, d'envisager les rapports qui se tissent, dans sa production littéraire, entre lecture et écriture, eu égard aux noüveaux barèmes d'une "modernité " en formation.

Le rapport du texte à sa lecture est l'un des plus solides qu'aient défini les écrivains de la modernité québécoise. Ceux-ci énoncent très tôt la velléité de doter le Québec d'une tradition de lecture ${ }^{13}$. La section "Les inédits" de La Barre du jour témoigne de cette intention. Cependant, chez les écrivains de la modernité, et chez Nicole Brossard en particulier, la lecture est bientôt canalisée vers l'écriture, et le désir absolu du texte dévié vers celui de son propre texte. On peut dire qu'en cela la démarche de Nicole Brossard s'apparente d'assez près à celle du lecteur tel que le définit Barthes dans Critique et Vérité ${ }^{14}$. Toute écriture semble ainsi prête à se mirer dans sa propre lecture, que celle-ci soit fantasmée (texte du désir ou désir du texte) ou programmée par divers artifices sémiologiques; corollairement, toute lecture pàraît devoir aboutir à un texte, comme si le texte était l'unique destin de la lecture. Dans un numéro de La Nouvelle Barre du jour au titre éloquent, L'Écriture comme lecture, Brossard évoque la relation ambiguë entre ces deux activités:

En pleine lecture, nous entendons des murmures, des suppliques, des cris; nous entendons une voix qui cherche son horizon. [...]

qui révèle le livre ultérieur, ponctuation qui témoigne d'autant plus du rôle spécifique et indispensable des revues dans l'histoire des écritures. , Double Impression, p. 7.

12. Nicole Brossard, "L'épreuve de la modernité ou/et les preuves de la modernité ", La Nouvelle Barre du jour, no 90-91, mai 1980, p. 55-68.

13. Dans une entrevue accordée à Voix \& Images en 1985, Marcel Saint-Pierre explique que l'aspect - exotique et régional. du titre de la revue, La Barre du jour, - signale une certaine façon de valoriser un patrimoine littéraire tombé en désuétude parce qu'on ne le rééditait pas et surtout l'intention de se doter d'une tradition de-lecture. Joseph Bonenfant et André Gervais, .Ce que pouvait être, ici, une avant-garde. Entrevue avec Nicole Brossard, Roger Soublière et Marcel SaintPierre., Voix E Images, vol. X, n' 2, hiver 1985, p. 69.

14. Roland Barthes, Critique et Vérité, Paris, Seuil, 1966, 78 p. 
Parmi la rhétorique, la logique des sens, les paradoxes et la sensation du devenir, nous avançons dans l'invention des formes ${ }^{15}$.

Comme souvent, chez Brossard, l'ambiguité tient à l'utilisation des ressources grammaticales et syntaxiques de la langue. Elle est liée à l'utilisation de ce que l'auteure a elle-même nommé un «joual syntaxique (par opposition au joual lexical des écrivains partipristes); ici le nous, qui paraît désigner une communauté de lecteurs, englobe peut-être aussi le producteur du texte que nous lisons; ainsi, la "voix qui cherche son horizon - peut à la fois être celle de l'auteur et celle du lecteur que nous sommes, qui cherche à identifier sa propre voix dans le texte lu ou qui cherche à poser sa voix quelque part dans les marges du texte; de même, l'ainvention des formes" peut renvoyer aussi bien aux formes du texte souche qu'aux formes que nous devons inventer pour parler de ce texte ou avec lui. On le voit: énonciateur et énonciataire(s), écriture et lecture permutent, se brouillent; néanmoins, le texte désénoncé profite d'abord au lecteur.

Ce n'est sans doute pas un hasard si la conscience de n'avoir rien de particulier à dire ${ }^{16}$ (il faut, affirme Soublière, :Axer l'effort sur le langage et non sur ce qu'on veut dire ${ }^{17}$ ) et l'hypostase de la lecture coïncident chez Nicole Brossard. Le texte brossardien participe résolumént d'une herméneutique qui, le plus souvent, s'éprouve comme délit d'interprétation, transgression des codes linguistiques, intellectuels et sociaux (les oukases du patriarcat, par exemple): le "texte", remarque l'auteure, "ressemble à un délit politique dont personne n'ose revendiquer la "paternité". Texte de filiation et texte d'affiliation, il s'affirme comme texte choisi ${ }^{18}$. " Après 1975, le texte met un peu plus souvent en scène, au moins sous la forme d'une simple nomination, le réseau des solidarités textuelles qui le fondent: y sont convoqués ici et là les travaux théoriques et littéraires des principales féministes américaines.

Foncièrement comprébensive et non judicative (pour reprendre les catégories de Jurt ${ }^{19}$ ), la lecture brossardienne se veut une lecture

15. Nicole Brossard, - Certains mots ', La Nouvelle Barre du jour, $n^{\circ}$ 157, 1985, p. 38-39.

16. Rien à exprimer, à tout le moins, d'une conscience particulière et irremplaçable: "J'ai dit "impression de vide", car le texte, on le sait, résume; il se résume autour de quelques mots: ville, sexe, texte, corps, désir, graphie, œeil (celui du cinéma, celui de la photo). Le texte est idéogramme. C'est en quelque sorte parce qu'il résume (il n'est pas bavard), qu'il réduit et qu'il va au plus court, qu'il donne cette impression de faire le vide ou de faire le fou (ce qui concernerait ses excès de vitalité). - Nicole Brossard, .L'épreuve de la modernité [...]., loc. cit., p. 62-63.

17. Joseph Bonenfant et André Gervais, loc. cit., p. 79.

18. Nicole Brossard, -La tendance , Liberté, vol. XIX, n 3 (111), mai-juin 1977, p. 41.

19. Joseph Jurt, -L'esthétique de la réception. Une nouvelle approche de la littérature? ?, Lettres romanes, vol. XXXVII, n 3, août 1983, p. 199-220. 
totale, ouverte à tout ce qu'elle saisit intuitivement comme immédiatement moderne, d'une modernité a-historique, synchronique - qui n'est donc pas inscrite dans une histoire des avant-gardes ${ }^{20}$. Pour Brossard, il semble bien que le texte n'ait pas à penser une théorie du moderne (ou du postmoderne), puisqu'il est lui-même, et dans l'immédiat, sa propre théorie. C'est pourquoi la définition de la modernité n'est pas un enjeu majeur de la lutte des agents à l'intérieur du champ de l'avant-garde; on s'accommode assez bien, somme toute, d'un consensus fondé sur des postulats implicites, ce dont témoignent plusieurs documents rassemblés dans le dossier $B J / N B J$ de la revue Voix et Images (par exemple, l'entrevue avec les fondateurs de la revue ${ }^{21} \mathrm{et}$ les lettres de refus).

Avec l'avènement de la módernité qu'incarne globalement la nouvelle écriture, la littérature québécoise passe le seuil décisif que la littérature française avait franchi un siècle plus tôt. Ce qu'Antoine Compagnon dit de la modernité du $\mathrm{XIX}^{c}$ siècle français s'applique parfaitement au cas du Québec: “[...] avec l'avènement de la "modernité", la distinction du présent et du passé s'évanouit elle-même dans l'éphémère ${ }^{22_{w}}$; dans le contingent, pourrait-on ajouter, puisque le fétiche "modernité " devient la matière de textes qui l'exhibent de façon toujours unique, toujours nouvelle. *Aussi la modernité est-elle une épreuve du sens, une mise à l'épreuve de soi dans l'expérience du langage", écrit Nicole Brossard dans un texte intitulé "Exactement là $23_{\text {n. }}$ " Mise à l'épreuve de soi ", "expérience du langage": la modernité apparaît ici, tout au plus, comme un label (un *trade mark *) apposé à

20. Roger Chamberland montre bien que, contrairement aux professeurs d'université qui ont tendance à inscrire toute modernité en cours dans une histoire littéraire des modernités ou une histoire institutionnelle des avant-gardes, la modernité de La Nouvelle Barre du jour et des Herbes rouges est une modernité a-historique qui, - délaissant toute conscience historique du phénomène, est définie de façon indistincte par plusieurs écrivains qui l'ont chargée d'un pouvoir expressif nouveau en la réduisant au champ du présent, de l'actuel et, l'identifiant avec le Zeitgeist, l'ont prise comme donnée de base dans le jugement de toute production poétique. - (op. cit., f. 63)

21. Entrevue où transparaît, c'est le moins qu'on puisse dire, une certaine désinvolture quant aux critères définitoires de la modernité - Brossard: :Corps, textes et sexes étaient nos petits fétiches -; Saint-Pierre: *Avec les numéros 9 et 10, la revie trouva sa couleur littéraire. L'appareil formalisant s'éclairait [...]. La revue avait atteint autour de ce moment ce qu'on peut appeler son "trade mark" '; Soublière: - Comme on savait ce qu'on ne voulait pas, il était facile de dire pourquoi on refusait un texte. Mais pourquoi disait-on oui à un texte? Concrètement, parce que c'était du jamais vu. - Joseph Bonenfant et André Gervais, loc. cü., p. 68-85.

22. Antoine Compagnon, Les Cinq Paradoxes de la modernité, Paris, Seuil, 1990, p. 25.

23. Nicole Brossard, „Exactement là , La Nouvelle Barre du jour, $\mathrm{n}^{\circ}$ 141, 1984, p. 29. 
une "recherche" à caractère essentiellement empirique et individuel. Dans les faits, le flottement dans les conceptions de la modernité ne pouvait avoir pour résultat qu'un certain éclectisme - sans doute fécond - des pratiques formalistes, de sorte que parler d'une influence déterminante, "coûteuse ${ }^{24}$, de Nicole Brossard, comme on le fait très souvent, paraît constituer un abus de langage.

Le formalisme de Nicole Brossard, on l'aura deviné, n'est en rien un formalisme doctrinaire. Bien au contraire, les modalités de la réalisation formelle de l'œuvre se réajustent dans chaque texte particulier, au point que l'écriture s'énonce avant tout dans son programme. Louise Dupré remarque à juste titre que, chez Brossard,

L'écriture se voit constamment prise dans l'écart entre la réalité de sa pratique et l'utopie de son énoncé. Et c'est cet espace à combler qui devient le moteur de l'écriture, qui l'actionne, met les mots en mouvement ${ }^{25}$.

Et elle poursuit:

Chez Nicole Brossard, le dire et le faire s'entrechoquent, se' déconcertent constamment, comme si l'écriture n'arrivait pas à cö̈ncider avec son objet $^{26}$.

Le texte brossardien est l'esquisse d'un texte à faire, d'un absolu du texte - d'un texte à venir, selon la formule de Blanchot. Il est le texte et son commentaire. Et il n'est chargé, en définitive, que d'exprimer une déception du texte: "Si l'on peut affirmer que la textualité intégrale est un mythe, on peut cependant dire que la pratique textuelle a pour effet d'inciter à une approche réflécbie de l'écriture et de la lecture ${ }^{27}$." Cela ne veut pas dire que ces textes fassent l'objet d'une autothéorisation ou d'une autocritique dysphoriques; posé à l'horizon de toutes les pratiques textualisantes, le "texte absolu "se trouve, d'une certaine façon, à valider le texte donné à lire, qui s'en avère l'anticipation. Par ailleurs, on ne peut pas dire que la critique parallèle des écrivains (par rapport à celle des professeurs d'université) ait déploré le décalage entre le projet de l'œuvre et l'œuvre à faire - entre le dire et le faire, suivant l'expression de Louise Dupré. On remarque, d'une part, que la majorité des œuvres importantes de

24. Louise Milot, *Nicole Brossard: une influence coûteuse,, Modernité/Postmodernité dans le roman québécois, op. cit., p. 77-86.

25. Louise Dupré, Stratégies du vertige, Montréal, Éditions du Remue-ménage, 1989, p. 86-87.

26. Ibid., p. 87.

27. Nicole Brossard, -Le réel et plus,, Choisir la poésie, Trois-Rivières, Écrits des Forges, 1986, p. 26. 
la période mettent en jeu un dispositif de légitimation intratextuelle, et, d'autre part, que les écrivains dits *de la' modernité * se sont légitimés entre eux dans leurs revues - la solidarité euphorique ${ }^{28} \mathrm{du}$ groupe prenant la forme d'une communauté émotionnelle. Visiblement agacé, Robert Melançon s'insurgeait dans Liberté contre ces deux formes complémentaires de légitimation - l'une, intratextuelle:

[...] l'œuvre de Nicole Brossard n'est qu'une somme d'informations sur l'œuvre de Nicole Brossard. Elle ne semble écrite que pour accrocher, pour évoquer sur un mode circulaire une œuvre qui n'existe pas autrement que dans l'annonce réitérée de sa propre imminence $[\ldots]^{29}$.

et l'autre, institutionnelle:

Nicole Brossard, co-fondatrice et co-directrice de La Barre du jour, fait l'objet d'un colloque organisé par cette revue où elle publie par ailleurs presque tous ses textes - et presque personne ne semble s'en étonner, cela va de soi, c'est parfaitement sérieux ${ }^{30}$.

C'est un fait que l'entreprise moderniste québécoise fait preuve, pour parler comme Roland Barthes, d'un singulier strabisme. Les producteurs et les agents régulateurs des pratiques *modernes" (fonctions habituellement cumulées, puisque les producteurs des formulaires discursifs et de la réceptión critique produisent aussi les "textes") appartiennent aux mêmes sphères, officient côte à côte chez les mêmes éditeurs et dans les mêmes comités de lecture, publient dans les mêmes revues. Jean Larose (1987) a longuement abordé cette question; nous n'insistons pas plus longtemps ${ }^{31}$.

Ce 'qu'il faut en revanche noter, c'est que Nicole Brossard produit aussi bien le formulaire discursif que la réception du texte:

on ne pose pas l'écriture en s'imaginant

que la réalité pourrait être autrement

plus douce. On les lie (c'est cette gerbe)

sous l'apparence de l'encre avec

des nouds baroques dans la gorge

épines bélier

cherchant le synchronisme

cailloux jardin

l'espace transitoire ${ }^{32}$

28. Solidarité qui a connu au moins une crise majeure en 1983-1984. Voir à cé propos Bernard Andrès, Écrire le Québec. De la contrainte à la contrariété, Montréal, XYZ, 1990, p. 165-176.

29. Robert Melançon, - Prière d'insérer ou pourquoi Nicole Brossard est un grand écrivain?, Liberté, vol. XXVI, n 5 (155), octobre 1984, p. 101.

30. Ibid., p. 102.

31. Jean Larose, La Petite Noirceur, Montréal, Boréal, 1987.

32. Nicole Brossard, Double Impression, op. cit., p. 96. 
- Dans ce court passage, semble s'élaborer une conception du texte comme espace transitoire (espace vide, mais énergétique, comme le blanc du centre et de la page) où l'imagination n'a plus nécessairement cours, l'encre, c'est-à-dire le trait, juxtaposant (la gerbe liée/le texte lu) des éléments hétéroclites, mais destinés à se rencontrer (le nœud, le baroque; "épines bélier ", "cailloux jardin "), pour créer une fulguration, une énergie synchrone, éphémère, mais néanmoins puis ${ }^{-}$ sante - et jouissive ${ }^{33}$. Ainsi donc, la forme du texte (elliptique, hétéroclite, baroque, etc.) est programmée et, du même coup, sa lecture. Le texte n'aborde aucun thème particulier; il n'explicite aucune " imagination. Il n'a d'autre visée que celle de s'énoncer dans sa textualité, fût-ce de manière sybilline ou détournée.

C'est parce que Nicole Brossard a surtout joué la carte d'une autolégitimation intratextuelle, qui passe avant tout par sa propre relecture, qu'elle a pu représenter un chef de file de la nouvelle écriture. C'est son relatif isolement qui lui a conféré ce titre et qui a pu contribuer à. la. constitution d'un "grand surmoi Brossard ", comme le signalait Normand de Bellefeuille. On constate en effet que l'œuvre de Brossard se tient à distance des autres œuvres de la modernité. Cette prise de distance tient, entre autres, au véritable barrage de gloses autolégitimantes qui la cerne; elle tient également à l'aspect résolu-, ment sec, ascétique de l'œuvre, du moins dans sa première phase. En somme, l'œuvre affiche spectaculairement les signes extérieurs de la rigueur; elle s'énonce de façon très intellectuelle, thématisant du reste un imaginaire de la sscience (l'hologramme, l'énergie, la géométrie, etc.). Et, bien que cette quvre se révèle tout compte fait assez profuse, elle paraît cultiver une morale de l'étique; à aucun moment, la jouissance du logos ne s'y éprouve ouvertement comme loghorrée. On est loin ici de la ferveur d'un Claude Beausoleil, ferveur qui s'exprime dans un vertige quantitatif:

La passion d'écriture traverse notre génération. Elle l'a affirmée par la quantité de sa production par rapport à celle des générations précédentes. Pour nous, il n'est pas question de prendre sa [sic] retraite très bientôt! [...] On est trop jeunes pour avoir des conclusions même un peu pessimistes sur ce qui est en train de se produire dans notre moder-. nité ou dans l'ensemble de la littérature québécoise qui, objectivement, quand on la compare aux corpus étrangers - au corpus français contemporain, par exemple - est strictement passionnante. [...] C'est absolument fantastique, la nouvelle littérature québécoise! [...] Nous

33. À propos de la figùre de l'énergie dans l'œuvre de Nicole Brossard, voir Pierre Nepveu, .Nicole Brossard: notes sur une écologie., La Nouvelle Barre du jour, $\mathrm{n}^{\circ}$ 118-119, novembre 1982, p. 139-144. 
vivons une situation stimulante, extrêmement riche. Une des spécificités dé notre modernité c'est qu'elle est allée très loin ${ }^{34}$.

Comparée à une consommation euphơrique du sens suscitée à la fois - et paradoxalement - par le réflexe consumériste et par les idéologies contre-culturelles (la valorisation de l'hédonisme, par exemple), la pratique de Nicole Brossard apparaît, dans un premier temps tout au moins, plus exigente, plus éthique, dans la mesure où elle est plus étique, pour reprendre un lieu commun barthésien de cette période. L'œuvre brossardienne s'inaugure en effet par une série de refus qui semblent en fonder la rigueur: refus de l'effusion et de l'expression, refus du biographisme, refus du beau style. S'avouant résolument moderne, cette œuvre signifie corollairement son désir de se retourner sur elle-même:

-Je ne suis pas un autre, dira le texte moderne après Rimbaud. J'existe, j'éclate la réalité et en voici les preuves. -Absolument moderne. va devenir - Résolument moderne?. L'expérience va se transformer en expérimentation: car le texte vise à la science. Il obsérve son fonctionnement. Il est à la fois objet et laboratoire. Découverte. Dé de hasard. Effet de rupture. Mais s'ajoute ici à • Résolument moderne • le caractère théorique de l'illisibilité 35 .

En dépit de' sa banalité, la confession est d'importance: la modernité du texte a ainsi pour corollaire (théorique?) son illisibilité. Par un réflexe typique, Brossard revendique ce qui, pour certains agents du champ, engagés dans une stratégie différente de distinction et de légitimation, peut apparaître comme une non-valeur. Crise du sens, crise du vers, crise du récit: la modernité se veut catastrophiste - et le texte moderne, impossible.

En dernière analyse, l'illisibilité revendiquée du texte brossardien entraîne, peut-être involontairement, une dépréciation de la lecture par autrui. Pour l'auteure, seule compte en définitive sa propre lecture (ou relecture) réfléchissante ${ }^{36}$ - lecture d'ailleurs inscrite dans le texte luimême, et qui non seulement l'interprète, mais en programme la réception critique (ce texte est moderne), à défaut de la réception effective (ce texte sera-t-il lu comme je désirerais qu'il le soit?). L'écrivain

34. Claude Beausoleil, cité par Jean Royer, loc. cit., p. 99-101.

35. Nicole Brossard, :L'épreuve de la modernité [...] , loc. cit., p. 58.

36. À Jack David, qui lui demandait si le blanc typographique, abondamment utilisé dans les poèmes, était dirigé vers le lecteur, Nicole Brossard répondait: * Non, c'est surtout pour moi que le blanc est là. Le lecteur, il va le prendre, mais tous les mots sont là pour moi. J Jack David, *Entrevie avec Nicole Brossard. Avant-postes - Out-Posts, Erin (Ontario), Presses Porcépic, 1978, p. 65-66. 
moderne est, faut-il le rappeler, celui qui se relit (à temps) ${ }^{37}$. D'ailleurs, jusqu'à un certain point, cette relecture de son texte se substitue chez Brossard à la lecture du texte d'autrui. En effet, et contrairement à ce qu'on pourrait croire à seulement prendre en considération le nimbe théorique dont s'entoure cette œuvre, les références explicites à d'autres textes, les citàtions et les commentaires demeurent rares. Dans les textes qui nous occupent surtout ici, textes qui ont en général pour fonction première d'expliciter le rapport de Brossard à l'écriture, les références aux théories littéraires sont peu nombreuses et s'avèrent, du reste, allusives; les rares citations sont tronquées ou de seconde main; les commentaires détaillés sont, à quelques exceptions près, inexistants. Parmi les théoriciens cités, Roland Barthes domine, mais on remarque que les ouvrages préférés de l'auteure ${ }^{38}$, L'Empire des signes, Le Plaisir du texte et Roland Barthes par Roland Barthes, ne constituent pas à proprement parler des travaux théoriques. En fait, 'c'est de manière souterraine, en vertu d'une intertextualité implicite, que les théories littéraires des années soixante et soixante-dix ont influencé Nicole Brossard ${ }^{39}$; à cet égard, Jean Larose a éloquemment témoigné de ce qu'un texte comme "Vague de précision" (pour' ne citer que cet exemple) devait aux séminaires d'Hélène Cixous à l'Université de Montréal.

En cela éminemment moderne, le texte brossardien est impérialiste. Il mime sans complexe les modèles européens de la modernité, qu'il s'agisse de Mallarmé, de Blanchot ou de Cixous; toutefois, cela s'accompagne d'une désarticulation des modèles, qui correspond à une appropriation et qui procure au surplus le sentimẹnt de la nouveauté. En effet, Brossard et la plupart des écrivains de sa génération ont posé une équivalence entre modernité et nouveauté. Or, comme le remarque Henri Meschonnic,

Le danger, pour le moderne, n'est pas la tradition. C'est le nouveau. Parce qu'il est ce qui lui ressemble le plus. Il tend à se faire prendre

37. Nicole Brossard note à ce sujet: -Je privilégiais, selon le principe de la lectureécriture, la relecture du texte au moment ou le texte s'écrit. - (Joseph Bonenfant et André Gervais, loc. cit., p. 79.) Et aussi: .[...]. mes manuscrits se corrigent au moment même où je les écris. [...]. C'est pourquoi, pendant que j'écris, je jette toujours un coup d'œil sur ce qui vient de s'écrire, ou peut-être est-ce simultané. . (Michel van Schendel et Jean Fisette, aUn livre à venir. Entrevue avec Nicole Brossard, Voix E Images, vol. III, $\mathrm{n}^{\circ} 1$, automne 1977, p. 4.)

38. Voir à ce sujet Jack David, op. cit.

39. Notons au passage que Nicole Brossard, à la différence de ses confrères Jan Stafford et Marcel Saint-Pierre de La Barre du jour, n'a pas fait partie de l'éphémère Groupe d'études théoriques - auquel appartenait par ailleurs France Théoret. 
pour lui, à se rendre indiscernable de lui. Et peut ne pas être lui. Si quelque chose est nouveau, comment savoir si c'est moderne? Et ce qui vient d'apparaître, en quel sens est-ce nouveau ${ }^{40}$ ?

On ne peut pas dire que ces questions agitent Nicole Brossard et les écrivains proches de la $B J / N B J$. À Jack David, qui proposait de rattacher son travail à une lignée issue de Marinetti et se poursuivant jusqu'aux lettristes, Nicole Brossard répliquait: "Moi, je dirai que mes poèmes sont complètement différents. Le seul lien qui puisse exister, ce serait un lien de révolte, celui de faire éclater les structures politiques, économiques, familiales ${ }^{41 . . ~ C e ~ q u i ~ e s t ~ p e u . ~ C o n c r e ̀ t e m e n t, ~ l a ~}$ valeur d'un texte soumis à la $B J / N B J$ tient au fait que * c'est du jamais vu *. L'autothéorisation et l'autolégitimation seront donc d'autant plus hâtives que le nouveau, par définition, ne dure pas, puisqu'il s'inscrit dans une logique du progrès qui l'historicise. Les écrivains qui gravitent autour de la $B J / N B J$ ont beau proclamer l'a-historicité de la modernité, ils l'engagent néanmoins dans la durée. Être d'avant-garde, c'est être en avance et rien de plus ("La Poésie ne rythmera plus l'action; elle sera en avant", écrivait Rimbaud). Cette littérature est condamnée par nature à sans cesse se recommencer, à réitérer sa nouveauté, à s'extasier de son propre avènement ${ }^{42}$.

Le rapport ambigu de Nicole Brossard aux textes du corpus moderne ${ }^{43}$ (rapport qui pousse l'auteure soit à ignorer ces textes, soit à y faire simplement allusion, soit encore à les mimer/incorporer) découle peut-être de l'appartenance des grands textes de la culture à un ensemble discursif étranger, c'est-à-dire français (pour l'essentiel) et masculin. "Forcément la femme qui a beaucoup lu s'insèrera plus facilement dans le jeu ", note Nicole Brossard dans "E muet mutant *

Toute son information se trouvant issue ou liée à une filiation d'œuvres inscrites dans un monde de pensée masculine, elle analyse, décode, joue, devient conséquente avec l'univers littéraire qu'elle a acquis. Elle s'illusionne à merveilles qui n'en sont pas toutes (sujet quand elle 'écrit, elle redevient vite objet le jour du lancement de son livre: elle devient l'objet rare, la caution, la preuve vivante que ça se peut une femme qui pense (le p'tit québecois millionnaire) ${ }^{44}$.

et elle reprend:

40. Henri Meschonnic, Modernitié modernité, Lapasse, Verdier, 1988, p. 77.

41. Jack David, op. cit., p. 64.

42. La neutralité des premiers recueils de Nicolè Brossard nous semble constituer une variante de ce rituel d'autocélébration. Le style figé, hiératique, convient parfaitement à l'édification d'une stèle.

43. Ne parlons pas des textes anciens, dont il est très peu question chez elle.

44. Nicole Brossard, *E muet mutant , La Barre du jour, n 50, hiver 1975, p. 15. 
Cetté femme qui lit, lit toujours à moitié, c'est-à-dire qu'elle doit s'épargner de sa condition, le consentement. Elle lit dans l'à peu près qui la rejoint du discours masculin 45 .

Lire, c'est donc participer à une culture étrangère sinon ennemie', cette culture patriarcale qui opprime le discours féminin. Après le tournant féministe de 1975 , Nicole Brossard va tenter de cerner l'enjeu de la lecture en termes de sexe (biologique) et de sexualité (lesbienne). À cette époque, pour l'auteure, il ne s'agit peut-être plus tant d'écrire pour une lecture, comme l'avait recommandé François Charron, que pour une lectrice.

Ce revirement dans la pensée de Nicole Brossard aura bien sûr une incidence sur ses lectures. À titre de modèles, vont succéder aux représentants de la nouvelle critique française les écrivaines féministes et/ou lesbiennes, pour la plupart québécoises ou anglo-saxonnes: Louky Bersianik, Djuna Barnes, Natalie Barney, Mary Daly, par exemple. Ce qui ne veut pas dire que l'écriture brossardienne s'ouvre largement aux autres écritures: celle-ci, en effet, reste d'abord et avant tout autoréférentielle. Après 1975, donc, là dérive - métaphore chère à Brossard - est continentale: vers le continent américain et vers le "continent-femmes". Évidemment, l'émergence d'un sujet féministe a aussi des répercussions sur l'écriture elle-même. Une pensée-femme cherche désormais à s'incarner dans un sujet-femme. Aspirer à une pensée féministe, c'est en quelque sorte désirer un recommencement de la pensée et de l'écriture. D'où il provient que la pensée féministe est, forcément, inaugurale. Si bien que les références aux grands textes masculins, déjà peu nombreuses avant 1975, se raréfient considérablement, et que l'entreprise brossardienne continue à se réclamer d'une logique intrinsèque, questionnant ses assises et tentant d'assurer sa propre validité comme écriture, et spécialement comme écriture féminine:

Et toujours cette écriture qui s'acharne sur le sens, renvoit au premier sens: suite à mon désir, suite à mon sexe. Je ne suis ni plus ni moins qu'un texte dira le texte mais ceci dit, toujours ramené au corps de l'auteur que je' suis; le taisant, le citant ou le rappelant ${ }^{46}$.

Sans aller ici jusqu'à conclure, avec Louise Milot, que le virage féministe de Nicole Brossard a lourdement hypothéqué la modernité littéraire québécoise (laquelle, dans sa première formulation, était de toute façon à boút de souffle), on est forcé de reconnaître que le

45. Ibid., p. 22.

46. Nicole Brossard, :La tendance, op. cit., p. 43. 
retour en force d'un contenu référentiel féministe a singulièrement infléchi la trajectoire et l'écriture de cette auteure. L'atténuation du caractère autoréférentiel du texte au profit d'un contenu explicitement féministe a sans doute contribué à réduire le rôle du processus de relecture dans l'écriture et, ainsi, à limiter les effets de miroir et de transparence qui avaient jusque là si profondément marqué le travail de Brossard.

En définitive, le projet brossardien consiste à la fois à écrire pour soi sa propre lecture de son texte et à écrire pour la lecture de l'autre, en prenant soin de guider cette lecture. Dans les deux cas, il est question, avant tout, d'écrire ce qui donne à lire. Ce n'est pas un hasard si l'un des principaux lieux communs de cette période consiste à désigner le processus de création comme un processus de lectureécriture. De fait, la circularité de la consommation des textes modernes - ces textes destinés aux seuls pairs et sympathisánts est reproduite dans les textes eux-mêmes, qui se lisent en même temps qu'ils s'écrivent, qui tiennent essentiellement à l'écriture de cette lecture et qui ne se tournent vers le lecteur que pour prévoir ou pour programmer sa lecture. L'un des traits distinctifs des textes formalistes produits après 1965 au Québec réside peut-être moins dans des caractéristiques formelles communes que dans une semblable mise en forme (en formulaire) des conditions de la lecture. Cette hypothèse demanderait bien sûr à être mise à l'épreuve. Cela dit, il est clair que, dans les textes de la modernité, la problématique de la lecture fait l'objet d'une véritable saturation théorique. Les "fictions/théories" ont pour objet, entre autres, de transformer une lecture disons hypothétique (le défaut de lecture, c'est-à-dire le défaut de réception ou d'interprétation) en lecture théorique - lecture balisée, idéalement compétente et informée, chargée de restituer l'intenté textuel.

Dans la mesure où Nicole Brossard est d'abord à l'écoute de son propre texte, la tradition littéraire ne s'y inscrit pas ostensiblement. Ses lectures transparaissent assez peu dans son travail, sinon sous forme d'allusions. C'est que le texte brossardien est davantage tourné vers sa réception et son devenir que vers ses origines et ses influences. Comme toute modernité, celle de Brossard revêt un aspect prophétique; elle est obsédée par sa propre révélation. Nous avons montré que, par certains côtés, la démarche de Brossard visait à recommencer la littérature; par conséquent, ón ne s'étonnera pas que le recommencement de l'écriture implique de facto celui de la lecture. Il semble, au total, que le projet initial de La Barre du jour, qui avait consisté pour une part à définir une tradition de lecture, se soit perdu en chemin, victime des nouveaux enjeux de la modernité. 